\title{
NOTATION SYSTEMS AND AN EFFECTIVE FIXED POINT PROPERTY ${ }^{1}$
}

\author{
WILLIAM E. RITTER ${ }^{2}$
}

1. Introduction. The notion of a "Gödel numbering" for partial recursive functions as in Rogers [4] is generalized to that of a "notation system." The relations of derivability among the semi-effective numberings extend to reducibilities among notation systems, and the familiar partial orders of recursive function theory are obtained. The basic result of Rogers [4] on the isomorphism of Gödel numberings is shown to hold for certain notation systems with an "effective fixed point property" which generalizes that property of the standard numbering of the partial recursive functions expressed by Kleene's Recursion Theorem.

2. Notation systems. Let us call a triple $(A, \alpha, P)$ a concrete notation system if $A$ is a nonempty set of natural numbers (nonnegative integers) and $\alpha$ is a function with domain $A$ and range $P$. (We will occasionally refer to such an $\alpha$ as a "notation system.") Associated with $\alpha$ is a relation $\sim_{\alpha}$ of synonymity, given by

$$
x \sim_{\alpha} y \equiv_{d f} x, y \in A \& \alpha(x)=\alpha(y) .
$$

Similarly, an abstract notation system is a pair $(A, \sim)$, where $\sim$ is an equivalence relation on the nonempty number-set $A$.

Example 1. $(N,=)$ and $(N, i, N)$, where $i$ is the identity function on $N=\{0,1,2, \cdots\}$, are abstract and concrete systems, respectively, in the above sense. In both cases, all natural numbers are "names" (and the objects named, in the latter case).

EXAmple 2. $\left(N, \lambda n W_{n}, \Pi_{1}^{1}\right)$ is a notation system for the class $\Pi_{1}^{1}$ of number sets definable with one universal function quantifier in the analytic hierarchy of Kleene, where

$$
W_{n}=\left\{x \in N \mid\left(\forall \alpha \in N^{N}\right)(\exists y \in N) T_{1}^{\alpha}(n, x, y)\right\} .
$$

Here the predicate $T_{1}^{\alpha}$ is defined in Kleene [2], and $\lambda n \phi(n)$ is the function $\phi$ given by the functional abstraction operator $\lambda$ of Church.

Received by the editors May 5, 1965.

1 Most of the results of this paper were first obtained in the first chapter of the author's doctoral dissertation at the Massachusetts Institute of Technology under the direction of Professor Hartley Rogers, Jr.

2 Research supported in part by a grant from the National Science Foundation. 
Example 3. $\left(\theta,|\cdot|, \omega_{1}\right)$ is the Church-Kleene "system of notations" $S_{3}$ for the constructive ordinals, where $|e|$ is the ordinal "named" by $e \in \mathcal{O}$ and $\omega_{1}$ is the first nonconstructive ordinal. See Kleene [2].

3. Reducibilities among notation systems. Names in one notation system may correspond, by virtue of common denotation, or otherwise, to names in another. For example the "names" $0,1,2, \cdots$ of the system $(N, i, N)$ correspond naturally to $1,2,4, \cdots$ of the system $\left(\mathcal{O},|\cdot|, \omega_{1}\right)$ under the correspondence $x \leftrightarrow x_{\mathcal{\Theta}}$ (where $0_{\mathcal{O}}=1$ and $\left.(n+1)_{\mathcal{O}}=2^{n_{0}}\right)$.

More generally, we shall say that a (concrete) notation system $\boldsymbol{A}$ is (recursively) many-one reducible (via $f$ ) to the system $B$ if $f$ is a (total) recursive function such that $\alpha=\beta f$, where $A=(A, \alpha, P)$ and $B=(B, \beta, Q)$. Note that this requires that $A \leqq_{m} B$ (via $f$ ) in the usual sense of recursive function theory (i.e., $A=f^{-1}[B]$ ), and that $\alpha(x)$ $=\beta(f(x))$ for $x \in A$ (so also $P \subseteq Q$ ). Similarly we shall say that an abstract system $\left(A, \sim_{A}\right)$ is many-one reducible via $f$ to $\left(B, \sim_{B}\right)$ if for all $x, y \in N, x \sim_{A} y$ if and only if $f(x) \sim_{B} f(y)$. Note also that this requires $A \leqq_{m} B$ via $f$, since $x \in A$ if and only if $x \sim_{A} x$. (In the notation of Rogers [5], we would write $f\left(\sim_{A}\right) \subseteq \sim_{B}$.) Finally, if $A \leqq_{m} B$ via $f$, where $f$ can be taken to be one-one, then we say that $A$ is one-one reducible to $B$ and write $A \leqq{ }_{1} B$.

EXAMPLE 4 . Let $\phi_{0}, \phi_{1}, \phi_{2}, \cdots$ be the standard enumeration of all partial recursive functions (as in [4]), and define $\alpha(n)=\phi_{n}^{-1}[\theta]$ for each $n \in N$. The maximality of $\mathcal{O}$ in $\Pi_{1}^{1}$ can be expressed by the assertion that the system of Example 2 is reducible to $\alpha$ (and $\theta=W_{\text {a }}$ for some $e \in N)$.

If $A \varliminf_{r} B$ and $B \varliminf_{r} A$ (where $\leqq_{r}$ is one of $\leqq_{m}, \leqq_{1}$ ), we say that $A$ and $B$ are $r$-equivalent $\left(A \equiv_{r} B\right)$. If $A \leqq_{1} B$ via $f$, and the range of $f$ is $N$ (so $f$ is both one-one and onto, i.e., a recursive permutation), then $B \leqq_{1} A$ via $f^{-1}$, and we say that $A$ and $B$ are (recursively) isomorphic $(A \cong B)$. The $r$-equivalences are reflexive, symmetric, and transitive, and hence equivalence relations (in the usual sense). The reducibilities are themselves transitive and reflexive and well defined on their equivalence classes, which are thereby partially ordered. The usual construction of recursive function theory applies so that the $m$-order is actually an upper semi-lattice (e.g., in the concrete case take $(\alpha \bigvee \beta)(k)$ as $\alpha(i)$ if $k=2 i$ and $i \in A$ and as $\beta(j)$ if $k=2 j+1$ and $j \in B$, with domain $A \cup B$ ).

EXAMPLE 5. The fact that the operation of taking inverse images by partial recursive functions does not lead out of the class $\Pi_{1}^{1}$, together with the facts stated in Example 4, shows that the $\alpha$ defined there is in fact $m$-equivalent to the system of Example 2. 
Reducibilities among notation systems in addition to those above may be defined and studied. For example, a Turing reducibility for abstract systems could be defined in analogy with Turing reducibility for number sets (e.g., we might put $\left(A, \sim_{A}\right) \leqq T\left(B, \sim_{B}\right)$ if $\sim_{A}$ is recursive in $\sim_{B}$ ). Certain classes $C$ of number-theoretic functions yield a $C$-reducibility $\leqq{ }^{C}$ provided that $i \in C$ and $C$ is closed under composition. Considered here are the special cases $C=G R$ (the class of general recursive functions), $C=G R_{1}$ (the one-one recursive functions), and $C=\mathcal{G}$ (the group of recursive permutations).

4. The effective fixed point property. The system of Example 2 (which represents the "standard Gödel numbering" of the sets in the class $\Pi_{1}^{1}$ ) has a special property whose generalization to other notation systems is useful. Namely, corresponding to every recursive function $f$ there is a number $n$, which depends effectively on (a Gödel number for) $f$, such that $W_{n}=W_{f(n)}$ (one form of the Recursion Theorem of Kleene).

In general, a notation system $A$ has the effective fixed point property $(A \in \mathcal{F})$ if corresponding to every recursive function $g$ there is a number $n_{0}$, depending effectively on $g$, such that either both $n_{0}$ and $g\left(n_{0}\right)$ fail to be in $A$, the domain of $A$, or else $n_{0} \sim g\left(n_{0}\right)$ according to the synonymity relation of $A$. If so, we say that $A \in \mathcal{F}$ via $f$, where $f$ is a recursive function with the property that if $e$ is an index (Gödel number) for $g$, then $f(e)$ will serve as $n_{0}$.

It is immediate that $\mathcal{F}$ is a recursively invariant property. For, if $A \cong B$ via $p$ and $A \in \mathcal{F}$ via $f$, then $B \in \mathcal{F}$ via $p f g$, where $g$ is a suitable recursive function which computes indices for $p^{-1} \phi p$ from indices for partial recursive functions $\phi$.

Example 6. The systems $(N,=)$ and $(N, i, N)$ are not in $\mathcal{F}$ (consider, e.g., the case where $g$ is the successor function).

EXAMPLE 7. Let Gödel numbers be assigned to Turing machines in an effective way so that all machines get numbers and yet the set $G$ of all such numbers is a proper subset of $N$, although recursive (cf. Davis [1, pp. 56-57]). Let $x \sim y$ in case both $x, y \in G$ and machines with numbers $x$ and $y$ both compute the same partial recursive function. Then $(G, \sim) \notin \mathcal{F}$, since we can find a recursive $g$ with $x \in G$ if and only if $g(x) \in N-G$. However, $\left(G^{*}, \sim^{*}\right) \in \mathcal{F}$ by a version of the Recursion Theorem for partial recursive functions, if we take $G^{*}=N$ and define $x \sim^{*} y$ as $f_{0}(x) \sim f_{0}(y)$, where $f_{0}$ is any (fixed) recursive function which has $G$ as its range.

5. Strengthening of reducibilities. In [4] Rogers shows that all "fully effective" numberings of the partial recursive functions are 
recursively isomorphic. In this section we shall adapt his arguments to show that, in certain cases, $m$-reducibility actually entails 1reducibility (and $m$-equivalence, 1 -equivalence). The basic construction needed for our theorems is the following:

Lemma. Suppose that $A \leqq_{m} B$ via $f$, where $f$ has infinite range, and that $A \in F$. Then there is a recursive function $\Phi$ of two variables whose values $\Phi(x, y)$ are in the domain $A$ of $A$ exactly when $x$ is, and if $x \in A$ then $x \sim \sim_{A} \Phi(x, y)$. Further, $f \Phi(x, y) \neq f \Phi(x, z)$ if $y \neq z$.

Proof. We define $\Phi$ by induction on its second argument. Clearly we may take $\Phi(x, 0)=x$ and suppose that $\Phi(x, z)$ has been defined for $z<y$. Now the function $g$ satisfying

$$
g(t)=\left\{\begin{array}{l}
x, \quad \text { if } f(t) \notin\{f \Phi(x, z) \mid z<y\}, \\
\mu w[f(w) \notin\{f \Phi(x, z) \mid z<y\}], \quad \text { otherwise, }
\end{array}\right.
$$

is recursive, since the infinitude of the range of $f$ makes effective the application of the least number operator $\mu$. Since $A \in \mathcal{F}$, there is a fixed point $n$ for $g$. Now we take $\Phi(x, y)$ as $g(n)$ or $n$, according as $f(n)$ is, or is not, in the set $\{f \Phi(x, z) \mid z<y\}$. By construction, all of $f \Phi(x, 0), f \Phi(x, 1), \cdots, f \Phi(x, y)$ are distinct. In case $f(n) \notin\{f \Phi(x, z) \mid z$ $<y\}$, we have $n=\Phi(x, y) \in A \leftrightarrow g(n)=x \in A$, and if $x \in A$ then also $\Phi(x, y) \in A$ (as we have just shown) and $\Phi(x, y)=n \sim_{A} g(n)=x$. In the opposite case $f(n) \in\{f \Phi(x, z) \mid z<y\}$, we have $f(n)=f \Phi(x, z)$ for some $z<y$, and thus $g(n)=\Phi(x, y) \in A \leftrightarrow n \in A \leftrightarrow f(n)=f \Phi(x, z) \in B$ $\leftrightarrow \Phi(x, z) \in A \leftrightarrow x \in A$, by the induction hypothesis (where $B$ is the domain of $B)$; and if $x \in A$, from $\Phi(x, z) \sim_{A} x, \Phi(x, y)=g(n) \sim_{A} n$, and $f(n)=f \Phi(x, z) \sim_{B} f(x)$, we conclude that $\Phi(x, y) \sim_{A} x$, again by the induction hypothesis. In either case $\Phi(x, y)$ is $A$-synonymous with $x$. Finally $\Phi$ is recursive since it can be effectively computed from $f$ and from $h$ such that $A \in \mathcal{F}$ via $h$.

Theorem 1. Under the hypotheses of the lemma, $A \leqq{ }_{1} B$.

Proof. Take $\Phi$ as given by the lemma and define $h$ by recursion so that $h(0)=0$ and $h(x+1)=\mu z[f \Phi(x+1, z)>f \Phi(x, h(x))]$. Now define $f_{1}(x)=f \Phi(x, h(x))$ for all $x \in N$. Clearly $f_{1}$ is monotone increasing, and it is easy to see that $A \leqq_{1} B$ via $f_{1}$.

\section{Theorem 2. If $A \leqq_{m} B$ and $B \in \mathcal{F}$, then $A \leqq_{1} B$.}

Proof. We have $B \varliminf_{m} B$ via $i$, the identity function on $N$. Apply the lemma (with $f=i$ and $A=B$ ) to obtain $\Phi_{1}$. Now if $A \leqq_{m} B$ via $g$, define $h$ so that $h(0)=0$ and $h(x+1)=\mu z\left[\Phi_{1}(g(x+1), z)>\Phi_{1}(g(x)\right.$, $h(x))]$. Then $A \leqq{ }_{1} B$ via $g_{1}$, where $g_{1}(x)=\Phi_{1}(g(x), h(x))$. 
ThEOREM 3. A notation system with the effective fixed point property is one-one equivalent precisely to those systems with which it is manyone equivalent.

Proof. Suppose that $A \leqq_{m} B$ via $f, B \leqq_{m} A$ via $g$, and $A \in F$. Then $A \equiv_{1} B$ by Theorems 1,2 , provided that $f$ has infinite range. Suppose, then, that the range of $f$ is finite, and take some fixed $x_{0} \in A$. Then the set $A_{0}=\left\{x \in A \mid x \sim_{A} x_{0}\right\}$ is recursive, since $B_{0}=f\left[A_{0}\right]$ is finite and $A_{0}=f^{-1}\left[B_{0}\right]$. Now it is clear that we must have $A=A_{0}=N=B_{0}=B$, and $A \cong B$ via every recursive permutation; for otherwise a recursive function $h$ which interchanges points in $A_{0}$ and $N-A_{0}$ can have no $A$-fixed point, contradicting the fact that $A \in \mathcal{F}$. (The burden of this proof was to show, in effect, that the analogue of Rice's Theorem (cf. $\left[1\right.$, p. 174]) holds for all systems in $F_{\text {.) }}$

6. Isomorphism of notation systems. The analogy with Rogers [4] is completed by the observation that the technique of Myhill [3] applies to yield isomorphism for 1-equivalent concrete notation systems. However, an example shows that such is not the case for abstract systems.

THEOREM 4. A concrete notation system with the effective fixed point property is isomorphic to any system with which it is many-one equivalent.

Sketch of PRoof. We may suppose, by Theorem 3 , that $A \leqq_{1} B$ via $f$ and $B \leqq_{1} A$ via $g$, where $f, g$ are one-one recursive functions. The main idea is to show that appropriate "finite correspondences" $\phi$ between $A$ and $B$ can be satisfactorily extended to permutations. Such a $\phi$ is of the form $\left\{\left\langle x_{0}, y_{0}\right\rangle,\left\langle x_{1}, y_{1}\right\rangle, \cdots,\left\langle x_{n}, y_{n}\right\rangle\right\}$, is a one-one function on its domain $D$, and is such that $\alpha\left(x_{i}\right)=\beta\left(y_{i}\right)$ for $0 \leqq i \leqq n$. We can effectively extend $\phi$ to a longer $\phi^{\prime}=\phi \cup\{\langle x, y\rangle\}$, where either $x$ is any arbitrary number not in $D$ and $y$ is $f(x)$ or some $f\left(x_{i}\right)$, or else $y$ is an arbitrary element in the complement of the range of $\phi$ and $x$ is $g(y)$ or some $g\left(y_{i}\right)$. By starting with the empty correspondence and alternately extending the range and domain of the correspondence, one arrives inductively at the required permutation.

EXAMPLE 8. Let $\phi_{n}$ be the partial recursive function with index $n$ (as in Example 4). Rogers calls a partial recursive function $\Phi$ universal [5] if $\phi_{x}=\lambda y \Phi f(x, y)$, for all $x$, where $f$ is some recursive function of two variables. Theorem 4 applies to yield the main result of $[5$, Theorem 2, p. 41], viz. for every pair $\Phi, \Psi$ of universal functions there is a recursive permutation $p$ with $\Phi=\Psi p$ (so $\Phi$ is said to resemble $\Psi)$. For, if we consider the system $\left(D_{e}, \phi_{e}, R_{e}\right)$ associated with any $\phi_{e}$ (with domain $D_{e}$, range $R_{e}$ ), then $\phi_{e} \leqq_{m} \Phi$ via $\lambda y f(e, y)$ if $\Phi$ is universal 
via $f$, so universal functions (themselves considered as notation systems in this sense) are $m$-equivalent. But also any such $\Phi$ has the effective fixed point property, as follows. According to the Recursion Theorem (for the sequence $\phi_{0}, \phi_{1}, \cdots$ ), given any recursive $g$, the equation $\lambda y \Phi f(g(m), y)=\lambda y \Phi f(m, y)$ can be solved effectively for $m$. Hence, given a recursive $h$, if $g$ is chosen so that $\phi_{g(x)}=\lambda y \Phi h f(y, y)$, then $f(m, m)$ serves as a fixed point for $h$, and the result follows.

It is interesting to observe that the Myhill technique of Theorem 4 does not apply to abstract systems in general. For, consider the system $A$ with equivalence classes $\{0\},\{1,2\},\{3,4\}, \cdots$ and the system $B$ with classes $\{0,1\},\{2,3\},\{4,5\}, \cdots$; then $A \equiv_{1} B$ via the successor function $\lambda x(x+1)$ in both directions and yet $A, B$ are not isomorphic. Of course, here neither $A$ nor $B$ has the effective fixed point property. Nevertheless Theorem 4 fails for certain abstract systems with this property, as the following shows (as well as the fact that 1-equivalence does not preserve $\mathcal{F}$ for all abstract systems).

EXAMPLE 9. Let $A=(\mathcal{O}, \sim)$ be the abstract system corresponding to the concrete system of Example 3 for the constructive ordinals (where $x \sim y \leftrightarrow|x|=|y|)$. Define $L=O \cap\left\{3.5^{e} \mid e \in N\right\}$ and let $\sim_{L}$ be the restriction of $\sim$ to $L$; then $B=\left(L, \sim_{L}\right)$ is an abstract system for the limit ordinals in $\omega_{1}$. Now we have $A \equiv_{1} B$ and $B \in \mathcal{F}$, and yet it is not the case that $A \cong B$. For, $B \leqq{ }_{1} A$ via $f$, where $f(x)$ is $x$ if $x=3.5^{e}$ for some $e$, and $7^{x}$ otherwise; and $A \leqq{ }_{1} B$ via $h$ defined as follows. Let $p(x, 0)=x$, and $p(x, n+1)=2^{p(x, n)}$; then $\lambda n p(x, n)=\phi_{g(x)}$ for some oneone recursive function $g$ (where $\phi_{z}$ is as in Example 4) and we may take $h(x)=3.5^{o(x)}$. To show that $B \in \mathcal{F}$, let any recursive $f$ be given and consider a partial recursive $\psi$, where $\psi(e, t)$ is $\phi_{z}(t)$ if $f\left(3.5^{e}\right)=3.5^{z}$ and 0 otherwise. Then $\lambda t \psi(e, t)=\phi_{h(e)}$ for some recursive $h$. By the Recursion Theorem we have $\phi_{h(m)}=\phi_{m}$ for some $m$. Take $n=3.5^{m}$. It is straightforward to verify that when $f(n)=3.5^{z}$ then $n \sim_{L} f(n)$ or else both $n, f(n)$ fail to be in $L$, and when $f(n)$ is not of this form then both $n, f(n)$ fail to be in $L$. On the other hand, the function $f$ with $f(0)=1$ and $f(n+1)=2^{f(n)}$ has all its values in $\mathcal{O}$ but has no $\mathcal{\theta}$-fixed point, so $A$ does not have the effective fixed point property.

\section{BIBLIOGRAPHY}

1. M. Davis, Computability and unsolvability, McGraw-Hill, New York, 1958.

2. S. C. Kleene, Arithmetical predicates and function quantifiers, Trans. Amer. Math. Soc. 79 (1955), 312-340.

3. J. R. Myhill, Creative sets, Z. Math. Logik Grundlagen Math. 1 (1955), 97-108.

4. H. Rogers, Jr., Gödel numberings of partial recursive functions, J. Symbolic Logic 23 (1958), 331-341.

5. - On universal functions, Proc. Amer. Math. Soc 16 (1965), 39-44.

Dartmouth College and

UNIVERSITY OF WASHINGTON 